\title{
Correlation Analysis of 5-HTTLPR Polymorphism and Post-Traumatic Stress Disorder in Li and Han Nationalities of Hainan Province
}

\author{
Ning Bei', Min Guo², Ping Huang³, Juncheng Guo ${ }^{2}$, Xiangling Jiang2*', Hong Gao', Dengyi Long1 \\ ${ }^{1}$ Hainan Cadre Sanatorium, Haikou, China \\ ${ }^{2}$ Hainan General Hospital, Haikou, China \\ ${ }^{3}$ Hainan Hospital of University of South China, Haikou, China \\ Email: *g2002m@163.com
}

How to cite this paper: Bei, N., Guo, M., Huang, P., Guo, J.C., Jiang, X.L., Gao, H. and Long, D.Y. (2019) Correlation Analysis of 5-HTTLPR Polymorphism and PostTraumatic Stress Disorder in $\mathrm{Li}$ and Han Nationalities of Hainan Province. World Journal of Neuroscience, 9, 243-254. https://doi.org/10.4236/wjns.2019.94018

Received: August 19, 2019

Accepted: October 8, 2019

Published: October 11, 2019

Copyright $\odot 2019$ by author(s) and Scientific Research Publishing Inc. This work is licensed under the Creative Commons Attribution International License (CC BY 4.0).

http://creativecommons.org/licenses/by/4.0/

\begin{abstract}
Objective: To analyse the correlation of 5-HTTLPR gene polymorphism and PTSD in Li and Han nationalities of Hainan Province. Methods: Essen trauma inventory (ETI), clinician administered post-traumatic stress disorders scale, (CAPS), tower of Hanoi (TOH), wsiconsin card sorting test (WCST), trail making test (TMT) and wechsler adult intelligence scale revised China (WAIS-RC) were used to investigate patients with PTSD and healthy volunteers (control group). PCR and PPGE were used to detect the polymorphism of 5-HTTLPR gene and analyze its correlation with PTSD. Results: The ETI score, total PTSD score and TMT time of Li nationality patients with PTSD were significantly higher than those of Han nationality patients with PTSD, and the IQ of comprehension and operation in WAIS-RC was significantly lower than that of Han nationality patients with PTSD $(\mathrm{P}<0.05)$. The ETI score, total PTSD score and TMT time of 5-HTTLPR genotype in Li nationality and Han nationality patients with PTSD were significantly higher than those of LL genotype, and the perception, calculation and block diagram in WAIS-RC were significantly lower than those of LL genotype patients $(\mathrm{P}<$ 0.05). Conclusions: The SS genotype can increase the risk of PTSD in Li nationality and Han nationality people in Hainan. LL genotype is associated with PTSD of Li and Han nationalities in Hainan, and is an important protective factor for PTSD of Li and Han nationalities in Hainan.
\end{abstract}

\section{Keywords}

5-HTTLPR, Gene Polymorphism, PTSD, Li Nationality, Han Nationality 


\section{Introduction}

Post-traumatic stress disorder (PTSD) is a debilitating anxiety disorder common in emotional trauma patients [1]. Women are more likely to suffer from PTSD than men [2]. When PTSD patients have a high comorbidity with PTSD, PTSD can become a common disabling disease in early adulthood. PTSD can become a common disability disease in early adults [2] [3]. Patients often experience invasive memory, avoidance and arousal symptoms, repeated empirical memory, fear, and a series of persistent physical and behavioral sequelae [1] [2] [3] [4]. At present, due to the lack of clinical subjects and short follow-up time, little research is focused on the prevention, development and treatment of PTSD [5]. Due to the moderate heritability of PTSD, the cause is presumed to be related to complex gene-environmental effects [6] [7]. Some scholars further speculate that individual differences in genetic susceptibility may play a role in the progression of PTSD. The 5- and serotonin-transporter-linked polymorphic region (5-HTTLPR) polymorphism is A feasible way to find effective treatment for PTSD [8] [9]. The severity of PTSD symptoms has some ethnic differences in patients [10]. This study explored the differences between 5-HTTLPR polymorphisms and PTSD symptoms and other cognitive endophenotypes in $\mathrm{Li}$ and Han populations in Hainan Province in order to find protective factors for PTSD.

\section{Materials and Methods}

\subsection{Research Object}

308 patients with PTSD who were transferred from Hainan Province from October 2011 to July 2014 and were treated in Hainan Provincial People's Hospital were selected, including 167 cases (54.2\%) of Li nationality and 141 cases (45.8\%) of Han nationality; 188 males and 120 females; mean age (45.2 \pm 5.6 ) years old (45.8\%); Case inclusion criteria: 1) According to the unified diagnostic criteria (DSM-IV, PTSD) of 2 middle-level and above-mentioned psychiatrists with rich clinical experience; the diagnosis is PTSD [11] [12]; 2) Living in Hainan for more than 10 years, and three or more generations of the same ethnic group are unrelated to each other; 3) Age 30 to 60 years old; 4) The physical condition feels good. Case exclusion criteria: 1) Having a family history of genetic psychosis; 2) Those with consumptive diseases such as tumors; 3 ) Women during pregnancy. A total of 158 healthy volunteers who underwent physical and non-traumatic events at the same time and were examined at the Health Checkup Center of Hainan Provincial People's Hospital as a control group, including 91 males and 67 females, with an average age of $(45.3 \pm 5.1)$ years. The study was approved by the hospital ethics committee and supervised by the hospital ethics committee. All subjects in this study were informed consent and signed informed consent.

\subsection{Methods}

\subsubsection{PTSD Indicator Detection}

Essen trauma inventory (ETI): Used to assess psychological trauma events and 
the psychological barriers they cause.

PTSD clinical assessment scale (CAPS scale, CAPS): 0 to 136 indicates the severity of PTSD symptoms in interview diagnosis [13].

Wechsler adult intelligence scale revised China (WAIS-RC).

Wisconsin card sorting test (WCST): Used to assess the tester's abstract thinking ability.

Trail making test (TMT): The ability to test the speed of psychology, attention and cognitive ordering, as well as spatial perception, eye-hand coordination, and flexible thinking.

Tower of Hanoi (TOH): If the subject is unable to resolve the problem within three chances, score 0 and the task stops.

\subsubsection{Gene Polymorphism Detection}

The subjects were enrolled in the morning, and the venous blood of the anterior elbow was $5-10 \mathrm{~mL}$. The EDTA was anticoagulated and sent to the Central Laboratory of Hainan Provincial People's Hospital, frozen at $-20^{\circ} \mathrm{C}$. Genomic DNA was extracted by whole-blood genomic DNA extraction kit (OMEGA, USA) by spin column method. The concentration and purity assays were in accordance with PCR amplification requirements. In this study, the internationally accepted primer sequences of the original literature (Table 1) were used, and the primers were synthesized by Shanghai Shenggong Bioengineering Technology Service Co., Ltd. PCR amplification reaction system a total of $50 \mu \mathrm{L}$, containing genomic DNA $1 \mu \mathrm{L}$, a pair of primers $1 \mu \mathrm{L}(10 \mu \mathrm{M})$, pfu enzyme $0.25 \mu \mathrm{L}$ $(5 \mathrm{U} / \mu \mathrm{L}), 10 \times$ Buffer $5 \mu \mathrm{L}(200 \mathrm{mM}$ Tris $\mathrm{HCl}, \mathrm{pH} 8.8 ; 100 \mathrm{mM} \mathrm{KCl} ; 20 \mathrm{mM}$ MgS04; $160 \mathrm{mM}$ (NH4) 2S04; 1\% Triton, $1 \mathrm{mg} / \mathrm{mL}$ (BSA), dNTPs $1 \mu \mathrm{L}(10 \mathrm{mM}$ ), deionized water $40.75 \mu \mathrm{L}$. PCR amplification cycle parameters: predenaturation at $98^{\circ} \mathrm{C}$ for 3 minutes, denaturation at $95^{\circ} \mathrm{C}$ for 1 minute, annealing at $60^{\circ} \mathrm{C}$ for 45 seconds, $72^{\circ} \mathrm{C}$ for 55 seconds, a total of 35 cycles, and then extended at $72^{\circ} \mathrm{C}$ for 8 minutes. The PCR kit was purchased from Shanghai Jierui Biological Engineering Co., Ltd. The PCR product was sent to Shanghai Shenggong Bioengineering Technology Service Co., Ltd. for sequencing.

\subsubsection{Statistical Analysis}

Experimental data were processed using SPSS 21.0 statistical software (SPSS Inc., Chicago, IL, USA). The normal distribution measurement data were expressed as mean \pm standard deviation. The variance was tested by homogeneity. The variance was compared between the two groups. The $t$ test was used. The variance or the non-conformity of the normal distribution was tested by Wilcoxon rank sum test. The count data is expressed by the composition ratio or rate, and the chi-square test is used. The genetic locus Hardy-Weinberg equilibrium test was performed using the $\chi^{2}$ test. The relative risk of genotypes is expressed by odd ratio (OR) and 95\% confidence interval (CI). The frequency distribution between the two groups was tested by chi-square test. Influencing factors of occurrence PTSD analysed by Logistic regression. $\mathrm{P}<0.05$ was considered statistically significant. 
Table 1. PCR primer sequences.

\begin{tabular}{llll}
\hline SNP & \multicolumn{2}{c}{ Sequence } & Anealing Temperature \\
\hline \multirow{2}{*}{ 5-HTTLPR } & Forward: & 5'-GGCGTTGCCGCTCTGAATGC-3' & $60^{\circ} \mathrm{C}, 45 \mathrm{~min}$ \\
& Reverse: & 5'-GAGGGACTGAGCTGGACAACCAC-3’ & \\
\hline
\end{tabular}

\section{Results}

\subsection{Comparison of General Data of Study Subjects}

Among the 167 patients with Li nationality PTSD, 98 were male and 69 were female, with an average age of $(45.4 \pm 5.3)$ years; Among the 141 Han patients with PTSD, 87 were male and 54 were female, with an average age of (44.9 \pm 5.8$)$ years; Of the 158 controls, 91 were male and 67 were female, with an average age of $(45.3 \pm 5.1)$ years. There were no significant differences in the mean age, gender composition, marital status, and education level among the three groups (all P > 0.05). The ETI score, PTSD total score, TMT time, TOH planning time and execution time, and WCST errors of Li PTSD patients and Han PTSD patients were significantly higher than those of the control group, WAIS-RC comprehension, similarity, wooden figure, object. The patchwork and operation IQ and TOH total scores were significantly lower than the control group; The ETI score, PTSD total score and TMT time of Li PTSD patients were significantly higher than those of Han PTSD patients. The comprehension and operational IQ of WAIS-RC were significantly lower than those of Han PTSD patients (there was a statistical significance $(\mathrm{P}<0.05)$. See Table 2 .

\subsection{Typing Analysis of 5-HTTLPR Gene Polymorphism}

The 5-HTTLPR gene can be detected by PCR amplification of 528 bp and 484 bp. The gene amplification products are divided into three genotypes: SS (484 bp/484bp), SL (484 bp/528bp) and LL (528 bp/528bp).

\subsection{5-HTTLPR Genotype and Allele Distribution}

The Hardy-Weinberg equilibrium fit was tested by the SHESIS software in the selected 158 healthy control groups. The results of the analysis showed that there was no significant difference between the theoretical frequency and the observed frequency of each allele distribution $(\mathrm{P}>0.05)$, the study sample selected in this experiment is a random population that meets the Hardy-Weinberg equilibrium.

There was no significant difference in the distribution of 5-HTTLPR genotypes and alleles between the PTSD patients and the control group (all $\mathrm{P}>0.05$ ). The distribution of 5-HTTLPR genotypes and alleles was significantly different between Han PTSD patients and control groups: SS genotype increased the risk of PTSD in Han population compared with LL genotype $(\mathrm{OR}=2.157,95 \% \mathrm{CI}=$ 1.098 - 4.237, $\mathrm{P}=0.024)$; compared with the $\mathrm{L}$ allele, the $\mathrm{S}$ allele increased the risk of PTSD in the Han population $(\mathrm{OR}=1.456,95 \% \mathrm{CI}=1.045-2.207, \mathrm{P}=$ 0.026). See Table 3. 
Table 2. Comparison of general data of study subjects.

\begin{tabular}{|c|c|c|c|c|c|c|}
\hline Item & $\begin{array}{l}\text { Control group } \\
\quad(\mathrm{n}=158)\end{array}$ & $\begin{array}{l}\text { Li PTSD Group } \\
\quad(\mathrm{n}=167)\end{array}$ & $\begin{array}{l}\text { Han PTSD group } \\
\quad(\mathrm{n}=141)\end{array}$ & $\mathrm{P}^{*}$ & $\mathrm{P}^{\#}$ & $\mathrm{P}^{\&}$ \\
\hline Average age (years) & $45.3 \pm 5.1$ & $45.4 \pm 5.3$ & $44.9 \pm 5.8$ & 0.917 & 0.492 & 0.434 \\
\hline Gender (male/female) & $91 / 67$ & $98 / 69$ & $90 / 51$ & 0.843 & 0.271 & 0.356 \\
\hline Marital Status (already/not) & $111 / 47$ & $101 / 66$ & $87 / 54$ & 0.064 & 0.119 & 0.826 \\
\hline Education level (year) & $10.9 \pm 2.6$ & $10.6 \pm 2.6$ & $10.6 \pm 2.5$ & 0.239 & 0.214 & 0.930 \\
\hline \multicolumn{7}{|l|}{ ETI score } \\
\hline Intrusion symptoms & $3.13 \pm 0.93$ & $5.98 \pm 2.45$ & $4.97 \pm 1.89$ & $<0.001$ & $<0.001$ & $<0.001$ \\
\hline Avoiding symptoms & $3.63 \pm 1.15$ & $6.27 \pm 1.99$ & $4.67 \pm 1.57$ & $<0.001$ & $<0.001$ & $<0.001$ \\
\hline Highly alert symptoms & $3.49 \pm 1.08$ & $5.20 \pm 2.09$ & $4.70 \pm 1.42$ & $<0.001$ & $<0.001$ & 0.016 \\
\hline Separation symptoms & $2.53 \pm 0.90$ & $4.40 \pm 1.63$ & $3.46 \pm 1.08$ & $<0.001$ & $<0.001$ & $<0.001$ \\
\hline PTSD total score & $15.14 \pm 6.13$ & $23.84 \pm 8.25$ & $19.54 \pm 6.29$ & $<0.001$ & $<0.001$ & $<0.001$ \\
\hline \multicolumn{7}{|l|}{ WAIS-RC } \\
\hline Knowledge & $10.67 \pm 1.75$ & $10.23 \pm 2.98$ & $10.52 \pm 2.58$ & 0.106 & 0.564 & 0.355 \\
\hline Comprehension & $11.78 \pm 2.10$ & $9.86 \pm 2.47$ & $10.74 \pm 2.16$ & $<0.001$ & $<0.001$ & 0.001 \\
\hline arithmetic & $10.30 \pm 2.19$ & $9.89 \pm 2.82$ & $9.99 \pm 2.32$ & 0.138 & 0.224 & 0.738 \\
\hline similarity & $11.08 \pm 1.75$ & $10.34 \pm 2.62$ & $10.43 \pm 2.52$ & 0.003 & 0.009 & 0.761 \\
\hline Digital breadth & $11.15 \pm 2.08$ & $10.44 \pm 3.56$ & $10.80 \pm 2.95$ & 0.029 & 0.242 & 0.130 \\
\hline vocabulary & $10.30 \pm 2.19$ & $10.10 \pm 2.64$ & $10.54 \pm 2.14$ & 0.440 & 0.349 & 0.109 \\
\hline Number symbol & $10.46 \pm 1.46$ & $10.11 \pm 2.63$ & $10.13 \pm 2.62$ & 0.109 & 0.138 & 0.944 \\
\hline Picture fills & $9.02 \pm 1.08$ & $8.59 \pm 2.56$ & $8.80 \pm 2.23$ & 0.054 & 0.276 & 0.450 \\
\hline Wooden block diagram & $10.78 \pm 2.10$ & $9.57 \pm 2.15$ & $9.97 \pm 1.47$ & $<0.001$ & $<0.001$ & 0.065 \\
\hline Images arrangement & $10.11 \pm 2.82$ & $9.62 \pm 2.49$ & $9.78 \pm 2.18$ & 0.096 & 0.257 & 0.559 \\
\hline Object patchwork & $11.98 \pm 1.79$ & $9.83 \pm 2.18$ & $10.10 \pm 1.98$ & $<0.001$ & $<0.001$ & 0.266 \\
\hline Language IQ & $101.37 \pm 15.01$ & $98.76 \pm 10.92$ & $99.43 \pm 10.23$ & 0.073 & 0.193 & 0.584 \\
\hline Operational intelligence & $101.58 \pm 10.00$ & $95.89 \pm 10.48$ & $98.76 \pm 13.25$ & $<0.001$ & 0.038 & 0.035 \\
\hline Total IQ & $102.80 \pm 10.64$ & $97.59 \pm 9.71$ & $99.16 \pm 9.42$ & $<0.001$ & 0.002 & 0.151 \\
\hline \multicolumn{7}{|l|}{ WCST } \\
\hline Number of categories & $4.11 \pm 0.93$ & $3.90 \pm 0.92$ & $3.91 \pm 1.03$ & 0.093 & 0.079 & 0.931 \\
\hline Number of errors & $13.44 \pm 4.32$ & $18.80 \pm 4.45$ & $19.04 \pm 4.25$ & $<0.001$ & $<0.001$ & 0.641 \\
\hline Correct number & $32.16 \pm 6.20$ & $30.90 \pm 6.03$ & $31.18 \pm 4.27$ & 0.064 & 0.119 & 0.649 \\
\hline Number of persistent errors & $4.70 \pm 2.13$ & $5.02 \pm 2.37$ & $4.88 \pm 2.52$ & 0.192 & 0.515 & 0.588 \\
\hline Random error number & $8.25 \pm 3.50$ & $8.63 \pm 3.02$ & $8.38 \pm 2.64$ & 0.299 & 0.734 & 0.438 \\
\hline \multicolumn{7}{|l|}{ TMT } \\
\hline A time (seconds) & $44.01 \pm 9.54$ & $53.83 \pm 12.07$ & $51.16 \pm 10.57$ & $<0.001$ & $<0.001$ & 0.042 \\
\hline B time (seconds) & $65.65 \pm 10.13$ & $79.60 \pm 8.97$ & $75.79 \pm 9.00$ & $<0.001$ & $<0.001$ & $<0.001$ \\
\hline \multicolumn{7}{|l|}{$\mathrm{TOH}$} \\
\hline Planning time & $5.81 \pm 2.21$ & $7.28 \pm 2.32$ & $7.15 \pm 2.03$ & $<0.001$ & $<0.001$ & 0.614 \\
\hline Execution time & $18.03 \pm 6.05$ & $26.07 \pm 6.05$ & $24.93 \pm 6.96$ & $<0.001$ & $<0.001$ & 0.205 \\
\hline Total score & $54.69 \pm 8.55$ & $46.13 \pm 9.57$ & $47.15 \pm 8.66$ & $<0.001$ & $<0.001$ & 0.332 \\
\hline
\end{tabular}

Note: * indicates that the Li PTSD group is compared with the control group; \# indicates that the Han PTSD group is compared with the control group; \& indicates that the Li PTSD group is compared with the Han PTSD group. 
Table 3. Comparison of 5-HTTLPR genotype and allele frequency distribution between PTSD patients and control groups in Li and Han nationalities $[\mathrm{n}(\%)]$.

\begin{tabular}{|c|c|c|c|c|c|c|c|}
\hline SNP & $\begin{array}{l}\text { Control group } \\
\quad(\mathrm{n}=158)\end{array}$ & $\begin{array}{l}\text { Li PTSD Group } \\
\quad(\mathrm{n}=167)\end{array}$ & $\begin{array}{c}\text { Han PTSD } \\
\text { group }(\mathrm{n}=141)\end{array}$ & $\mathrm{P}^{*}$ & $\mathrm{OR}^{\star}(95 \% \mathrm{CI})$ & $\mathrm{P}^{\#}$ & $\mathrm{OR}^{\#}(95 \% \mathrm{CI})$ \\
\hline \multicolumn{8}{|c|}{ 5-HTTLPR } \\
\hline LL & $35(22.2)$ & $26(15.6)$ & $18(12.8)$ & Ref. & & Ref. & \\
\hline LS & $68(43.0)$ & $83(49.7)$ & $62(43.9)$ & 0.141 & $0.640(0.352-1.161)$ & 0.089 & $1.773(0.912-3.446)$ \\
\hline SS & $55(34.8)$ & $58(34.7)$ & $61(43.3)$ & 0.325 & $0.732(0.392-1.364)$ & 0.024 & $2.157(1.098-4.237)$ \\
\hline $\mathrm{LS}+\mathrm{SS}$ & $123(77.8)$ & $141(84.4)$ & $123(87.2)$ & 0.129 & $0.648(0.369-1.137)$ & 0.004 & $1.944(1.045-3.619)$ \\
\hline $\mathrm{L}$ & $138(43.7)$ & $135(40.4)$ & $98(34.8)$ & Ref. & & Ref. & \\
\hline S & $178(56.3)$ & $199(59.6)$ & $184(65.2)$ & 0.401 & $0.875(0.641-1.195)$ & 0.026 & $1.456(1.045-2.027)$ \\
\hline
\end{tabular}

Note: SNP: Single Nucleotide Polymorphisms; OR: Odds Ratio; 95\% CI: 95\% confidence intervals; Ref: Reference. * indicates that the Li PTSD group is compared with the control group; \# indicates that the Han PTSD group is compared with the control group.

\subsection{Relationship between 5-HTTLPR Polymorphism and PTSD}

Among Li patients with PTSD: compared with patients with 5-HTTLPR LL genotype, patients with SS genotype had significantly higher ETI scores, PTSD total scores, and TMT time, and comprehension and arithmetic and block diagrams and operations in WAIS-RC IQ was significantly reduced (both $\mathrm{P}<0.05$ ). Among Han patients with PTSD: compared with patients with 5-HTTLPR LL genotype, patients with SS genotype had significantly higher ETI scores, PTSD total scores, and TMT time, and comprehension and arithmetic and block diagrams and operations in WAIS-RC IQ was significantly reduced (both $\mathrm{P}<0.05$ ). See Table 4.

\subsection{Logistic Regression Analysis of Various Factors}

The relationship between age, sex, marital status, education level, 5-HTTLPR gene and PTSD of Li and Han were analyzed by two-category logistic regression analysis, with or without disease as the dependent variable. The analysis results showed that: The LL genotype of 5-HTTLPR was significantly correlated with PTSD of both Li and Han $(\mathrm{P}<0.05)$. There was no significant correlation between age, gender, marital status and education level and PTSD between Li and Han $(\mathrm{P}>0.05)$. See Table 5.

\section{Discussion}

5-HTTLPR is located about $1 \mathrm{~kb}$ upstream of the transcription start site of the 5-HTT gene, which generates a long allele (L, containing 16) due to the insertion/deletion of a $44 \mathrm{bp}$ base fragment in 6 - 8 repeat elements. Repeat units) and short alleles (S, containing 14 repeat units) [14]. Studies have found that 5-HTTLPR genotype may play an important role in the development of PTSD and the severity of symptoms, and 5-HTTLPR polymorphism may increase the risk of PTSD [4]. Xu GZ et al. reported that the 5-HTTLPR S allele can increase the risk of PTSD in combat veterans [15]. The S allele in 5-HTTLPR is associated 
Table 4. Relationship between 5-HTTLPR gene polymorphism and PTSD in Li and Hannationalities.

\begin{tabular}{|c|c|c|c|c|c|c|}
\hline \multirow{3}{*}{ Item } & \multicolumn{3}{|c|}{$\mathrm{Li}$} & \multicolumn{3}{|c|}{ Han } \\
\hline & \multicolumn{3}{|c|}{ 5-HTTLPR } & \multicolumn{3}{|c|}{ 5-HTTLPR } \\
\hline & $\mathrm{LL}(\mathrm{n}=26)$ & LS $(\mathrm{n}=83)$ & SS $(\mathrm{n}=58)$ & $\operatorname{LL}(\mathrm{n}=18)$ & LS $(n=62)$ & SS $(n=61)$ \\
\hline \multicolumn{7}{|l|}{ ETI score } \\
\hline Intrusion symptoms & $6.46 \pm 3.05$ & $5.83 \pm 2.21$ & $5.98 \pm 2.49^{*}$ & $3.72 \pm 1.07$ & $4.79 \pm 2.15$ & $5.53 \pm 1.48^{\#}$ \\
\hline Avoiding symptoms & $6.31 \pm 1.78$ & $6.15 \pm 1.90$ & $6.43 \pm 2.20^{*}$ & $3.66 \pm 1.33$ & $4.05 \pm 1.08$ & $5.59 \pm 1.59^{\#}$ \\
\hline Highly alert symptoms & $4.89 \pm 2.37$ & $5.25 \pm 1.85$ & $5.23 \pm 2.32^{\star}$ & $3.67 \pm 1.28$ & $4.32 \pm 1.39$ & $5.44 \pm 1.06^{\#}$ \\
\hline Separation symptoms & $4.42 \pm 1.45$ & $4.41 \pm 1.59$ & $4.38 \pm 1.80^{\star}$ & $2.56 \pm 1.04$ & $3.15 \pm 0.96$ & $4.05 \pm 0.88^{\#}$ \\
\hline PTSD total score & $23.38 \pm 10.25$ & $24.06 \pm 7.97$ & $23.74 \pm 7.80^{\star}$ & $15.44 \pm 7.46$ & $17.69 \pm 4.78$ & $22.62 \pm 5.92^{\#}$ \\
\hline \multicolumn{7}{|l|}{ WAIS-RC } \\
\hline Knowledge & $9.62 \pm 3.41$ & $10.61 \pm 2.88$ & $9.99 \pm 2.90$ & $10.44 \pm 2.50$ & $10.40 \pm 2.92$ & $10.67 \pm 2.26$ \\
\hline Comprehension & $9.42 \pm 2.37$ & $9.85 \pm 2.34$ & $10.05 \pm 2.70^{*}$ & $12.11 \pm 3.27$ & $12.08 \pm 0.87$ & $8.99 \pm 1.22^{*}$ \\
\hline Arithmetic & $9.46 \pm 2.76$ & $10.17 \pm 2.96$ & $9.67 \pm 2.65$ & $11.56 \pm 2.68$ & $10.92 \pm 2.19$ & $8.57 \pm 1.41^{\#}$ \\
\hline Similarity & $10.54 \pm 2.50$ & $10.07 \pm 2.56$ & $10.62 \pm 2.82$ & $10.50 \pm 2.46$ & $10.10 \pm 2.86$ & $10.74 \pm 2.15$ \\
\hline Digital breadth & $10.73 \pm 3.46$ & $10.45 \pm 3.46$ & $10.28 \pm 3.79$ & $11.11 \pm 2.81$ & $10.84 \pm 2.93$ & $10.67 \pm 3.04$ \\
\hline Vocabulary & $9.39 \pm 2.43$ & $10.16 \pm 2.59$ & $10.33 \pm 2.76$ & $10.78 \pm 1.99$ & $10.39 \pm 2.40$ & $10.62 \pm 1.92$ \\
\hline Number symbol & $10.15 \pm 2.24$ & $10.20 \pm 2.75$ & $9.97 \pm 2.67$ & $10.50 \pm 2.75$ & $10.05 \pm 2.60$ & $10.11 \pm 2.63$ \\
\hline Picture fill & $8.62 \pm 2.93$ & $8.60 \pm 2.50$ & $8.57 \pm 2.51$ & $9.16 \pm 2.43$ & $8.74 \pm 2.20$ & $8.75 \pm 2.23$ \\
\hline Wooden block diagram & $9.58 \pm 1.86$ & $9.64 \pm 2.22$ & $9.41 \pm 2.18$ & $11.06 \pm 1.11$ & $10.61 \pm 1.06$ & $9.00 \pm 1.34^{\#}$ \\
\hline Picture arrangement & $10.04 \pm 2.84$ & $9.51 \pm 2.54$ & $9.60 \pm 2.26$ & $10.17 \pm 2.09$ & $9.45 \pm 2.46$ & $10.00 \pm 1.88$ \\
\hline Object patchwork & $9.19 \pm 2.02$ & $9.93 \pm 2.15$ & $9.98 \pm 2.28$ & $9.72 \pm 1.60$ & $10.42 \pm 1.92$ & $9.88 \pm 2.13$ \\
\hline Language IQ & $99.81 \pm 12.65$ & $98.45 \pm 9.84$ & $99.19 \pm 11.74$ & $100.3 \pm 9.49$ & $98.68 \pm 11.58$ & $99.92 \pm 9.02$ \\
\hline Operational intelligence & $95.46 \pm 9.50$ & $96.86 \pm 10.33$ & $94.71 \pm 11.14^{\star}$ & $107.7 \pm 15.93$ & $102.1 \pm 11.76$ & $92.70 \pm 11.16^{\#}$ \\
\hline Total IQ & $98.54 \pm 10.98$ & $97.51 \pm 9.56$ & $97.28 \pm 9.49$ & $101.6 \pm 9.17$ & $98.40 \pm 10.50$ & $99.21 \pm 8.32$ \\
\hline \multicolumn{7}{|l|}{ WCST } \\
\hline Number of categories & $4.08 \pm 0.98$ & $3.80 \pm 0.88$ & $3.97 \pm 0.95$ & $4.06 \pm 1.00$ & $3.73 \pm 1.12$ & $4.05 \pm 0.92$ \\
\hline Number of errors & $18.92 \pm 4.85$ & $18.72 \pm 4.08$ & $18.86 \pm 4.85$ & $18.11 \pm 6.18$ & $18.21 \pm 1.90$ & $20.15 \pm 5.05$ \\
\hline Correct number & $31.81 \pm 4.85$ & $30.78 \pm 6.61$ & $30.66 \pm 5.67$ & $32.22 \pm 3.75$ & $30.29 \pm 4.67$ & $31.79 \pm 3.85$ \\
\hline Number of persistent errors & $4.07 \pm 1.96$ & $5.23 \pm 2.47$ & $5.16 \pm 2.34$ & $4.89 \pm 3.18$ & $4.94 \pm 2.30$ & $4.80 \pm 2.61$ \\
\hline Random error number & $8.23 \pm 2.88$ & $8.90 \pm 3.00$ & $8.41 \pm 3.11$ & $7.50 \pm 2.96$ & $8.50 \pm 2.71$ & $8.51 \pm 2.47$ \\
\hline \multicolumn{7}{|l|}{ TMT } \\
\hline A time (seconds) & $55.19 \pm 9.82$ & $53.35 \pm 12.65$ & $53.09 \pm 12.27^{*}$ & $48.89 \pm 15.90$ & $45.66 \pm 3.54$ & $57.43 \pm 10.18^{\#}$ \\
\hline B time (seconds) & $81.23 \pm 7.98$ & $79.12 \pm 8.67$ & $79.57 \pm 9.83^{\star}$ & $71.50 \pm 7.46$ & $72.65 \pm 7.20$ & $80.26 \pm 9.18^{\#}$ \\
\hline \multicolumn{7}{|l|}{$\mathrm{TOH}$} \\
\hline Planning time & $7.50 \pm 2.05$ & $6.95 \pm 2.27$ & $7.64 \pm 2.48^{\star}$ & $7.17 \pm 1.98$ & $7.00 \pm 2.32$ & $7.30 \pm 1.74$ \\
\hline Execution time & $25.73 \pm 5.41$ & $26.48 \pm 5.99$ & $25.64 \pm 6.45$ & $21.94 \pm 7.79$ & $24.79 \pm 7.08$ & $25.95 \pm 6.43$ \\
\hline Total score & $46.73 \pm 8.08$ & $45.54 \pm 9.63$ & $46.71 \pm 10.18^{*}$ & $47.44 \pm 8.56$ & $46.48 \pm 9.90$ & $47.74 \pm 7.33$ \\
\hline
\end{tabular}

Note: * indicates the LL genotype of 5-HTTLPR in patients with PTSD in Li, $\mathrm{P}<0.05$; \# indicates the LL genotype of 5-HTTLPR in Han PTSD patients, $\mathrm{P}<$ 0.05 . 
Table 5. Logistic regression analysis.

\begin{tabular}{cccccccc}
\hline & & & & & \multicolumn{2}{c}{$95 \%$ CI for Exp (B) } \\
\cline { 5 - 7 } Factor & B & S.E. & Wald & sig. & Exp (B) & Lower & Upper \\
\hline Li & & & & & & & \\
age & 0.010 & 0.022 & 0.022 & 0.649 & 1.010 & 0.967 & 1.056 \\
gender & 0.481 & 0.276 & 0.276 & 0.082 & 1.618 & 0.942 & 2.780 \\
marital status & -0.337 & 0.291 & 0.291 & 0.247 & 0.714 & 0.404 & 1.262 \\
education level & -0.050 & 0.044 & 0.044 & 0.257 & 0.951 & 0.872 & 1.037 \\
5-HTTLPR & -0.865 & 0.305 & 0.305 & 0.005 & 0.421 & 0.232 & 0.765 \\
Han & & & & & & & \\
age & -0.009 & 0.023 & 0.157 & 0.692 & 0.991 & 0.948 & 1.036 \\
gender & 0.515 & 0.281 & 3.368 & 0.066 & 1.674 & 0.966 & 2.901 \\
marital status & -0.239 & 0.293 & 0.663 & 0.416 & 0.788 & 0.444 & 1.399 \\
education level & -0.051 & 0.048 & 1.112 & 0.292 & 0.950 & 0.865 & 1.045 \\
5-HTTLPR & -0.800 & 0.384 & 4.349 & 0.037 & 0.449 & 0.212 & 0.953 \\
\hline
\end{tabular}

with an increased risk of PTSD patients living in a criminal community, lacking social support and high unemployment, or experiencing child adversity and traumatic events [16]. The relationship between 5-HTTLPR polymorphism and negative life events and major depression in Chinese population has been elucidated. The 5-HTTLPR polymorphism increases the susceptibility of $20-29$ years to first-onset adolescents with major depressive disorder [17]. PTSD is a complex mental disorder that can occur in traumatic events such as car accidents, rape, combat exposure or natural disasters [18]. Post-traumatic stress disorder poses a serious health, social and economic burden on individuals and society as a whole [19]. 5-HTTLPR polymorphism plays an important role in the occurrence and treatment of post-traumatic stress disorder [4] [20] [21]. In order to investigate the effect of 5-HTTLPR gene polymorphism on PTSD, we have studied the PTSD patients of Li and Han nationality in Hainan Province. The results show that the ETI score, PTSD total score, TMT time and TOH planning time of PTSD patients in both $\mathrm{Li}$ and Han countries are found. The number of execution time and WCST errors were higher than those of the control group, and compared with the control group, the WAIS-RC similarity, block mode, understanding, object assembly and operation IQ of PTSD patients decreased, indicating that PTSD affects people indicating that PTSD affect the general behavioral abilities of people. At the same time, in this study, compared with Han patients with post-traumatic stress disorder, the ETI score, total post-traumatic stress disorder and TMT time of Li people with post-traumatic stress disorder were higher, but WAIS-RC understood and operated. IQ is lower. WCST showed an implementation flaw, the number of errors was used to assess post-traumatic 
stress disorder in prisoners of war, and more persistent WCST errors were observed in severe cases [22] [23]. The results of this study show more total errors in patients with post-traumatic stress disorder. Lower IQ and pre-traumatic education achievements play a crucial role in the post-traumatic stress disorder symptoms. Speech memory, working memory and attention performance, and speech intelligence are negatively correlated with symptoms of post-traumatic stress disorder [24]. In this study, the ETI score, PTSD total score, and TMT time of PTSD patients in Han and Li populations in Hainan Province were higher, but their understanding, operation IQ, and block mode were lower. In our study, 5-HTTLPR (VNTR) was divided into SS, SL and LL genotypes, and the 5-HTTLPR (VNTR) genotype distribution in the Li and Han ethnic groups was consistent with the Hardy-Weinberg equilibrium, indicating 5-HTTLPR (VNTR) can be used in subsequent experiments.SS genotype and $\mathrm{S}$ allele increase the risk of post-traumatic stress disorder. The results of the 5-HTTLPR genotype have three biallelic genotypes, namely SS, SL and LL. S homozygotes in PTSD are higher than L homozygotes and heterozygotes, and results from scholars such as Liu Y and Braithwaite EC. S homozygotes in PTSD are higher than L homozygotes and heterozygotes, which is consistent with the results of Liu Y, Braithwaite EC et al. [9] [25]. The genotypes of subjects from the Detroit Community Health Study were consistent with the Hardy-Weinberg balance and found that the SL genotype was most common among the three 5-HTTLPR biallelic genotypes [4]. This study showed that the SS genotype and S allele in 5-HTTLPR (VNTR) increased the risk of PTSD in the Han nationality in Hainan. As a $44 \mathrm{bp}$ insertion/deletion, the 5-HTTLPR polymorphism has two alternative gene variants, including the $S$ allele and the $L$ allele [26]. In many wound exposures, the $\mathrm{S}$ allele is a risk factor for PTSD, and the SS genotype also appears to be a specific risk factor for PTSD [4]. Hirohashi S et al. confirmed that SS genotype is a higher risk factor for PTSD in the case of high traumatic exposure [27], Patients with the $S$ allele in 5-HTTLPR had significantly worse PTSD symptoms than patients with homozygous L allele [21]. Similarly, the incidence of PTSD was found to be higher compared to L homozygotes and heterozygotes, and the addition of the 5-HTTLPR S allele promoted the severity of PTSD. S allele subjects showed a higher risk of PTSD symptoms than homozygous L allele subjects [21]. These findings are consistent with the results of this study. However, this study has certain limitations, and differences between groups may affect the 5-HTTLPR gene polymorphism. Therefore, this study is more meaningful for larger samples. Population-based differences, especially the degree of linkage disequilibrium between the measured polymorphism and other potential variations in the gene, may contribute to inconsistent findings. These findings open up new avenues for research that help to understand the relationship between genetic polymorphisms and post-traumatic stress disorder.

\section{Supporting Information}

Supported by Hainan Provincial Natural Science Foundation of China (No. 
818MS163) and Hainan Provincial Key Research and Development Program (No. ZDYF2018227).

\section{Conflicts of Interest}

The authors declare no conflicts of interest regarding the publication of this paper.

\section{References}

[1] Roth, T.L., Zoladz, P.R., Sweatt, J.D. and Diamond, D.M. (2011) Epigenetic Modification of Hippocampal Bdnf DNA in Adult Rats in an Animal Model of Post-Traumatic Stress Disorder. Journal of Psychiatric Research, 45, 919-926.

https://doi.org/10.1016/j.jpsychires.2011.01.013

[2] Chapman, C., Mills, K., Slade, T., et al. (2012) Remission from Post-Traumatic Stress Disorder in the General Population. Psychological Medicine, 42, 1695-1703. https://doi.org/10.1017/S0033291711002856

[3] Robinson, B.L. and Shergill, S.S. (2011) Imaging in Posttraumatic Stress Disorder. Current Opinion in Psychiatry, 24, 29-33. https://doi.org/10.1097/YCO.0b013e3283413519

[4] Walsh, K., Uddin, M., Soliven, R., Wildman, D.E. and Bradley, B. (2014) Associations between the SS Variant of 5-HTTLPR and PTSD among Adults with Histories of Childhood Emotional Abuse: Results from Two African American Independent Samples. Journal of Affective Disorders, 161, 91-96.

https://doi.org/10.1016/j.jad.2014.02.043

[5] Andero, R., Brothers, S.P., Jovanovic, T., et al. (2013) Amygdala-Dependent Fear Is Regulated by Oprl1 in Mice and Humans with PTSD. Science Translational Medicine, 5, 188ra73. https://doi.org/10.1126/scitranslmed.3005656

[6] Banerjee, S.B., Morrison, F.G. and Ressler, K.J. (2017) Genetic Approaches for the Study of PTSD: Advances and Challenges. Neuroscience Letters, 649, 139-146. https://doi.org/10.1016/j.neulet.2017.02.058

[7] Forresi, B., Caffo, E. and Battaglia, M. (2015) Gene Environment Interplays: Why PTSD Makes a Good Case for Gene-Environment Interaction Studies and How Adding a Developmental Approach Can Help. In: Martin, C., Preedy, V. and Patel, V., Eds., Comprehensive Guide to Post-Traumatic Stress Disorder, Springer, Cham, 1-13. https://doi.org/10.1007/978-3-319-08613-2_84-1

[8] Soliman, F. (2010) Variant Brain-Derived Neurotrophic Factor Val66Met Endophenotypes: Implications for Posttraumatic Stress Disorder. Annals of the New York Academy of Sciences, 1208, 150-157. https://doi.org/10.1111/j.1749-6632.2010.05722.x

[9] Liu, Y., Garrett, M.E., Dennis, M.F., et al. (2015) An Examination of the Association between 5-HTTLPR, Combat Exposure, and PTSD Diagnosis among U.S. Veterans. PLoS ONE, 10, e0119998. https://doi.org/10.1371/journal.pone.0119998

[10] Chen, T., Guo, M., Gao, Y.S., et al. (2014) A Comparative Study on the Levels of Serum Cytokines and Cortisol among Post-Traumatic Stress Disorder Patients of $\mathrm{Li}$ and Han Ethnicities in Hainan. National Medical Journal of China, 127, 2771-2774.

[11] Kilpatrick, D.G. (2013) The DSM-5 Got PTSD Right: Comment on Friedman (2013). Journal of Traumatic Stress, 26, 563-566. https://doi.org/10.1002/jts.21844

[12] Kilpatrick, D.G., Resnick, H.S., Milanak, M.E., et al. (2013) National Estimates of Exposure to Traumatic Events and PTSD Prevalence Using DSM-IV and DSM-5 
Criteria. Journal of Traumatic Stress, 26, 537-547. https://doi.org/10.1002/jts.21848

[13] Blake, D.D., et al. (1995) The Development of a Clinician-Administered PTSD Scale. Journal of Traumatic Stress, 8, 75-90. https://doi.org/10.1007/BF02105408

[14] Buyati, D., Adila Duolikun, Y., Nanzamu, Y., et al. (2012) 5-HTTLPR Gene Insertion/Deletion Polymorphism Study and Longevity Association Analysis in Hetian Uygur Elderly. Science and Technology Review, 26, 59-63.

[15] Xu, G.Z., Liu, Y., Zhang, Y., et al. (2015) Correlation between VEGFR2 rs2071559 Polymorphism and Glioma Risk among Chinese Population. International Journal of Clinical and Experimental Medicine, 8, 16724-16728.

[16] Telch, M.J., Beevers, C.G., Rosenfield, D., et al. (2015) 5-HTTLPR Genotype Potentiates the Effects of War Zone Stressors on the Emergence of PTSD, Depressive and Anxiety Symptoms in Soldiers Deployed to Iraq. World Psychiatry, 14, 198-206. https://doi.org/10.1002/wps.20215

[17] Zhang, K., Xu, Q., Xu, Y., et al. (2009) The Combined Effects of the 5-HTTLPR and 5-HTR1A Genes Modulates the Relationship between Negative Life Events and Major Depressive Disorder in a Chinese Population. Journal of Affective Disorders, 114, 224-231. https://doi.org/10.1016/j.jad.2008.07.012

[18] Su, Y.A., Jun, W., Lei, Z., et al. (2008) Dysregulated Mitochondrial Genes and Networks with Drug Targets in Postmortem Brain of Patients with Posttraumatic Stress Disorder (PTSD) Revealed by Human Mitochondria-Focused cDNA Microarrays. International Journal of Biological Sciences, 4, 223-235. https://doi.org/10.7150/ijbs.4.223

[19] Chen, Z.J., Zhao, H., He, L., et al. (2010) Genome-Wide Association Study Identifies Susceptibility Loci for Polycystic Ovary Syndrome on Chromosome 2p16.3, 2p21 and 9q33.3. Nature Genetics, 43, 55-59. https://doi.org/10.1038/ng.732

[20] Neumeister, A. (2013) The Role of BDNF-TrkB Signaling in the Pathogenesis of PTSD. Journal of Depression \& Anxiety, S4, 1-6. https://doi.org/10.4172/2167-1044.S4-006

[21] Young, K.A., Morissette, S.B., Jamroz, R., et al. (2017) 5-HTTLPR and DISC1 Risk Genotypes for Elevated PTSD Symptoms in US Military Veterans. World Psychiatry, 16, 109-110. https://doi.org/10.1002/wps.20359

[22] Kanagaratnam, P. and Asbjørnsen, A.E. (2007) Executive Deficits in Chronic PTSD Related to Political Violence. Journal of Anxiety Disorders, 21, 510-525. https://doi.org/10.1016/j.janxdis.2006.06.008

[23] Digangi, J.A., Kujawa, A., Aase, D.M., et al. (2017) Affective and Cognitive Correlates of PTSD: Electrocortical Processing of Threat and Perseverative Errors on the WCST in Combat-Related PTSD. Progress in Neuro-Psychopharmacology and Biological Psychiatry, 75, 63-69. https://doi.org/10.1016/j.pnpbp.2017.01.004

[24] Aupperle, R.L., Melrose, A.J., Stein, M.B. and Paulus, M.P. (2012) Executive Function and PTSD: Disengaging from Trauma. Neuropharmacology, 62, 686-694. https://doi.org/10.1016/j.neuropharm.2011.02.008

[25] Braithwaite, E.C., Ramchandani, P.G., O'Connor, T.G., et al. (2013) No Moderating Effect of 5-HTTLPR on Associations between Antenatal Anxiety and Infant Behavior. Journal of the American Academy of Child \& Adolescent Psychiatry, 52, 519-526. https://doi.org/10.1016/j.jaac.2013.02.010

[26] Plieger, T., Montag, C., Felten, A. and Reuter, M. (2014) The Serotonin Transporter Polymorphism (5-HTTLPR) and Personality: Response Style as a New Endophenotype for Anxiety. The International Journal of Neuropsychopharmacology, 17, 851-858. https://doi.org/10.1017/S1461145713001776 
[27] Hirohashi, S., Clausen, H., Nudelman, E.D., et al. (1986) A Human Monoclonal Antibody Directed to Blood Group I Antigen: Heterohybridoma between Human Lymphocytes from Regional Lymph Nodes of a Lung Cancer Patient and Mouse Myeloma. Journal of Immunology, 136, 4163-4168. 